\title{
Towards the numerical simulation of seismic excitation
}

Doz, G.N., Universidad Nacional de Tucumdn, Argentine

Riera, J.D.

Universidade Federal do Rio Grande do Sul, Porto Alegre, RS, Brazil

\begin{abstract}
Recent advances in the numerical simulation of the rupture process in solids, namely mode I fracture propagation, are extended to account for sliding with friction along preexisting fracture surfaces, with the final objective of analyzing seismic fault motions. The procedure is applied to the study of the resulting stick-slip motion of a prismatic block resting by gravity on a flat rigid surface. The result, although not yet confronted with experimental observations, suggests that the basic assumptions are correct and permits drawing a number of conclusions on the characteristics and evolution of stick-slip motion.
\end{abstract}

\section{INTRODUCTION}

The origin of seismic motions is attributed to a process of faulting and fracture, and/or to sudden relative movement of big portions of rock, caused by a state of stress that varies very slowly with time. It is clear then that progress in the understanding of rupture phenomena in solids, like fracture propagation and fracture induced vibrations, will contribute to explain and eventually quantify different aspects of the seismic excitation at sites of interest.

Initially, the possibility that earthquakes might be modeled as mode II (shear) fracture processes was considered. However, a close examination of the problem strongly suggests that, in materials with the properties of crystalline rock, mode II fracture does not exist. The initial crack orientation always coincides with the minimum principal strain direction, indicating a process of fracture in mode I. Based on their experiences with single-notched specimens, Arrea and Ingraffea (1982) and Ingraffea and Gerstle (1985) conclude that concrete fractures in mode I, even if a high mode II intensity factor is present. In 1985, Ingraffea and Panthaki reach the same conclusion by presenting finite element results for the double-notched shear beam tested by Bazant and Pfeiffer (1986), which reveal that failure must have occurred in mode $I$ also, in spite of, Bazant and Pfeiffer (1986) contention that failure was essentially of a shear type.

Rots and de Borst (1987) study numerically the single-notched beam tested by Arrea and Ingraffea (1982) and the double-notched beam studied by Bazant and Pfeiffer (1986). The finite elements analysis reveals that the mode I fracture energy was released much quicker than the mode II fracture energy in both cases, which suggests that fracture propagates predominantly in mode $I$.

For the mixed-mode fracture studies of Kobayashi et al (1985) using cracked-line wedge-loaded, double cantilever beam models and those of Jenq and Shah (1988) using 
three point bend, offset notched-beam specimens, it is found that the response could be adequately simulated using fracture-process zone (FPZ) models for mode I concrete fracture only, once the crack turns into a mode I propagation direction. Similar studies for rock materials are unknown to the authors, but it seems reasonable to assume that the behavior observed in concrete applies also to fracture in crystalline rock. Thus, it is herein accepted that the direction of crack propagation is perpendicular to the maximum principal stress (strain) directions. Consequently, the possibility of mode II crack propagation was ruled out in this study which, in addition to mode I fracture, considers the possibility of sliding along pre-existing fracture surfaces.

\section{FRICTION IN SOLIDS}

As explained above, seismic motions are regarded as caused by sliding of two rock volumes compressed against each other along preexisting fault surfaces, due to slowly increasing shear stresses. Initially, the two volumes are stuck against each other due to the presence of interface friction. Although this is one of the most complex phenomena of solids mechanics, it may be just mentioned in this paper. In fact, an important class of dynamic problems that includes such effects as frictional damping, dynamic sliding, stickslip motion, etc, is designated as dynamic sliding friction. The constitution of the material interface must be essentially stable; there is no marked penetration or normal plastic deformation of the interface, and from a global point of view, the frictional forces developed on the contact surface appear to depend on the sliding velocity of one surface relative to the other. When two surfaces in contact slide relative to each other, intermittent vibration can often be observed, especially at low driving velocities.

Blok (1940), analyzing experimental results of Bowden and Lebes (1939) and of Papenhuyzen (1938) concluded that the essential condition for the occurrence of stickslip motion is a decrease of the frictional force with increasing sliding speeds. Oden and Martins (1985) understand that stick-slip motion is a manifestation of dynamic instabilities inherent in the coupling of normal and tangential relative motions of contacting bodies. This phenomenon is not necessarily the result of a decrease in the coefficient of friction with changes in sliding velocity, and can be observed when the coefficient of friction is constant, as will be shown later, justifying the adoption of the simplest criteria to account for sliding.

\section{THEORETICAL MODEL}

The numerical model consists in the representation of solids as an array of normal and diagonal bars, linking lumped nodal masses (Riera et al, 1991). A dynamic analysis is performed, using explicit numerical integration in the time domain. At each step of integration a nodal equilibrium equation is solved by means of the central finite differences scheme:

$$
m u_{i}+c u_{i}=f_{i}
$$

where $\mathrm{m}$ denotes the nodal mass, $\mathrm{c}$ the damping constant, $u_{i}$ a component of the nodal coordinates vector and $f_{i}$ a component of the resulting forces at one nodal point (idirection), including restitutive (i..e. elastic), external and frictional forces. Dots denote time derivatives. The constitutive law allows for elasto-plastic behaviour, with provisions to account for fracture in mode I. In view of the many uncertainties existing in relation 
with sliding in rock, the interface constitutive relations are parsimoniously based on the assumption that the simple dry friction criterium applies.

\section{NUMERICAL RESULTS}

The sliding motion of a prismatic block resting on a rigid surface was studied first. Fig. 1 shows the model analyzed. Its dimensions and loading were chosen having a future experimental verification in mind. It consists of an homogeneous block of dimensions $1.0 * 0.5 * 0.1 \mathrm{~m}$ that rests on a rigid surface. The block is subjected to initial stresses due to its own weight, after which an horizontal motion with constant velocity of a nodal point on its left surface is specified. Although the capability of the model to handle complex material and interface constitutive relations is quite high, the results reported herein were obtained on the assumption that the material remains elastic. Thus, at low shear stress levels along the interface, stresses increase monotonically without any relative displacement along the interface. Eventually, the interface shear stress at a point exceeds the local shear strength, assumed to be equal to the normal stress times the local friction coefficient, and slip at a node occurs, which may propagate to the surrounding area, causing a sudden mean stress-drop associated with important displacements (see Fig. 2) Whenever this "seismic"event occurs, significant interface normal vibrations and separation during slip are observed, associated with oscillation of the normal stresses, which implies that the usual assumption of constant normal stress is not valid (see Fig. 3). A local decrease of normal stress is registered with no change in the coefficient of friction. These results are consistent with preliminary experimental work (Brune et al, 1993) that suggests that normal interface vibratory motion may be important. Moreover, Haskell (1964) suggests that normal interface vibrations must occur during an earthquake, which would be responsible for the yet unexplained high frequency $P$ waves radiation. A sample of the acceleration time-history at a location near the "fault" plane is shown in Fig. 4. Numerical simulation was performed for velocities of the control point in the range $0.55 .10^{-4}-0.25 .10^{-4} \mathrm{~m} / \mathrm{s}$, and for coefficients of interface friction equal to 1 to 6 , corresponding to angles of internal friction from $45^{\circ}$ to $80^{\circ} 30^{\prime}$. The stick-slip behaviour described in Figs. 2, 3 and 4 was observed for friction coefficients above 2. For low friction, some of the regions mentioned in section 5 are not identifiable.

\section{CONCLUSIONS}

On the basis of the numerical simulation studies described in section 4 , the following conclusions can be drawn:

a) peak accelerations near the slip zone vary linearly with the mean stress-drop

b) high frequency normal vibrations precede every important stick-slip, i.e., "seismic event"

c) four different stages may be distinguished in the global sliding processes:

1) a "build up" region in which tangential stresses increase without any motion between adjacent points on both fault surface

2) a region with distinct "seismic events" indicated at times denoted $E_{i}$ in Fig. 2, characterized by stress-drops varying between 10 and $50 \%$ of the mean shear stress

3) a region in which the shear stress still increases, accompanied by significant normal vibrations, and micro tremors characterized by small stress drops, of the order of a few percentual points of the mean shear stress, and

4) a region of steady state motion, in which the block slides with constant velocity plus internal vibrations of small amplitude. 


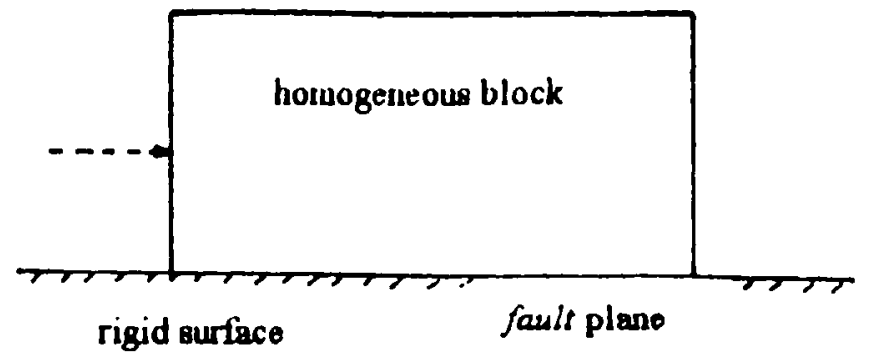

Fig. 1: Homogeneous block submeted to a prescribed displacement

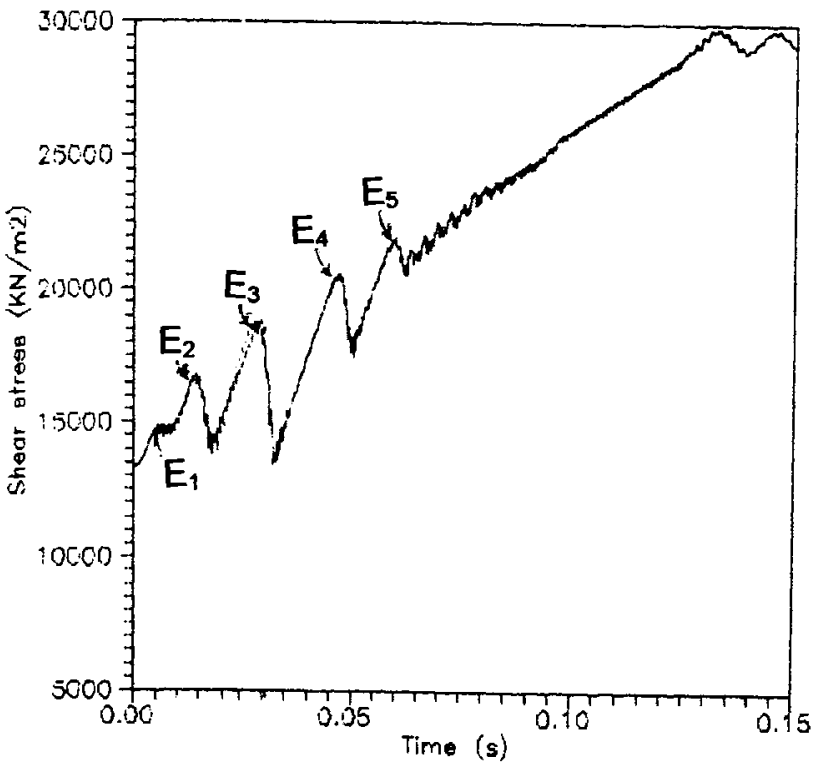

Fig, 2: Mean shear stress evolution 




Fig. 3: Normal stress evolution

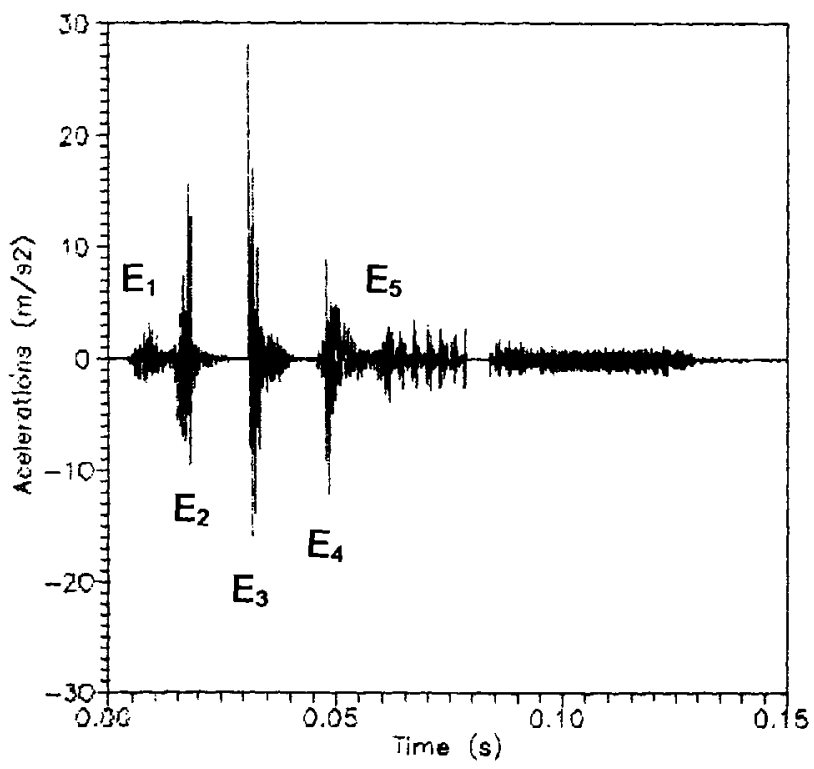

Fig. 4: Acceleration evolution 
Region 2 exists only when the friction coefficient exceeds a threshold value. It is suggested that the last part of region 3 and region 4 may correspond to what in Seismology, and obviously with other time and space scales, is designated as "creep sliding".

Another interesting feature of the analysis is that, although the interface contact is considered perfect, the friction coefficient constant and the block properties also constant, the predicted stick-slip process has an intrinsic random nature, i.e., it is very sensitive to the displacement rate and to the initial conditions, represented by the initial contact normal stresses. Note that the initial normal stress distribution is not uniform, depending on how the block is laid on the surface.

\section{AKNOWLEDGEMENTS}

This note was partly supported by CONICET (Argentine) and CNPq (Brazil)

\section{REFERENCES}

Arrea, M. \& Ingraffea, A.R., 1982. Mixed-mode crack propagation in mortar and concrete. Report No 81-1, Dept. of Struct. Engrg., Cornell Univ., Ithaca, NY, USA.

Bazant, Z. \& Pfeiffer, P.A., 1986. Shear fracture tests of concrete. Materiux et Constructions (RILEM) 19:111-121.

Blok, H., 1940. Fundamental mechanical aspects of boundary lubrication. S.A.E. J. 46(2), 54-68.

Bowden, F.P. \& Leben, L., 1939. The nature of sliding and the analysis of friction. Proc. Roy. Soc. London A 169, 371-391.

Brune, J., Brown, S. \& Johnson, P., 1993. Rupture mechanism and interface separation in foam rubber models of earthquakes: a possible solution to the heat flow paradox and the paradox of large over thrusts. Tectonophysics, 218:59-67.

Haskell, N., 1964. Total energy and energy spectral density of elastic wave radiation from propagating faults. Bull. Seismol. Soc. Am., 54:1811-1841.

Ingraffea, A.R. \& Gerstle, W., 1985. Nonlinear fracture models for discrete crack propagation. Application of Fracture Mechanics to Cementitious Composites, S.P. Shah ed., Martinus Nijhoff Publishers, Dordrecht, 247-285.

Ingraffea, A.R. \& Panthaki, M.J., 1985. Analysis of "shear fracture" tests of concrete beam. US-Japan Seminar on F.E. Analysis of Reinforced Concrete Struct.1, Tokyo, $71-91$.

Jenq, Y.S. \& Shah, S.P., 1988. Mixed-mode fracture of concrete. Int. J. of Fracture, $338 ; 123-142$.

Kobayashi, A.S. \& et al., 1985. Fracture process zones of concrete. Application of Fracture Mechanics to Cementitious Composites, S. Shah, ed., M. Nijhoff Pub., Dordrecht, 25-30.

Oden, J.T. \& Martins, J.A.C., 1985. Models and computational methods for dynamic friction phenomena. Computer Methods in Applied Mechanics and Engineering, 52:527 634 .

Papenhuyzen, P.J., 1938. Wrijvingsproeven in verband met het slippen van autobanden. De Ingenieur, 53, 75-81.

Riera, J.D. \& Rocha, M., 1991. A note on the velocity of crack propagation in tensile fracture. Revista Brasileira de Ciências Mecânicas, RBCM, Vol.XII, No 3, 217-240.

Rots, J.G. \& de Borst, R., 1987. Analysis of mixed-mode fracture in concrete. Journal of Engineering Mechanics, V.113, No 11. 